\title{
Applications de la dissolution complète du lait à la détermination de quelques activités enzymatiques
}

\author{
par \\ G. LINDEN, G. HUMBERT, R. DESNOUVEAUX \\ et Jocelyne PICARD*
}

\begin{abstract}
Rés u mé
Le mélange n-butylamine/cyclohexanone/triton X-100 (2:1:1) permet de dissoudre aisément et simultanément tous les constituants colloïdaux du lait. Ce solvant peut être utilisé dans la détermination d'activités enzymatiques susceptibles de libérer un produit de réaction absorbant entre 400 et $800 \mathrm{~nm}$ et qui reste stable à $\mathrm{pH} \mathrm{10-11.} \mathrm{On}$ commence par la réaction enzymatique classique que l'on bloque à un temps donné par l'addition de ce mélange dissolvant. Cette technique a notamment été appliquée avec succès à la mesure des activités phosphatasique, peroxydasique, protéinasiques et $\beta$-D-galactosidasique dans le lait et de quelques produits laitiers. Les tests de répétabilité ont montré que les protocoles proposés satisfont aux conditions requises par un laboratoire de contrôle ou d'analyse de l'industrie laitière.

\section{Mots clés}

Dissolution - Lait - Phosphatase alcaline - Peroxydase - Protéinases - $\beta$-Dgalactosidase.
\end{abstract}

Titre abrégé

Activités enzymatiques après dissolution du lait.

\section{S u m m a ry}

APPLICATIONS OF TOTAL DISSOLUTION OF MILK TO ENZYMIC ACTIVITIES DETERMINATIONS

A mixture of n-butylamine, cyclohexanone and triton X-100 (2:1:1) solubilizes the colloïdal components of milk. This solvent has been

* Service de Biochimie Appliquée, Université de Nancy I - 54506 Vandœuvreles-Nancy cedex. 
successfully used for some types of enzymic determination with release of products absorbing in the scale of 400 to $800 \mathrm{~nm}$, and stable at $\mathrm{pH}$ 10-11. One begins with the classical enzymic reaction which is stopped at a given time with the addition of the dissolving mixture. This technic was used i.e. for the measurement of phosphatasic, peroxydasic, proteinasic and $\beta$-D-galactosidasic activities in bovine milk. The repetability tests showed that these process would be satisfactory applied by control or analysis laboratories in the dairy industries.

Key words

Dissolution - Milk - Alkaline phosphatase - Peroxydase - Proteinases - $\beta$-Dgalactosidase.

Run title

Enzymatic activities in transparent milk.

\section{INTRODUCTION}

L'extrême turbidité du lait, due à la présence de micelles de caséines et de globules gras, rend impossible tout dosage des composants du lait par spectrométrie d'absorption moléculaire sans un traitement préliminaire de l'échantillon.

Bosset, Blanc et Plattner (1977 a, b) ont étudié la dissolution intégrale du lait au moyen de divers solvants mixtes pour permettre précisément de tels dosages. Ainsi, le mélange dissolvant eau/soude/nbutylamine fut appliqué avec succès au dosage des protéines totales du lait entier par la méthode dite du biuret (Bosset et Blanc, 1977).

En partant du même principe, nous avons mis au point, dans un précédent travail, un mélange dissolvant qui permet de déterminer directement l'activité de la phosphatase alcaline du lait (Linden et Paquet, 1981). Suite à ce développement, nous avons étudié la possibilité d'utiliser ce mélange pour la détermination d'autres activités enzymatiques dans le lait.

En premier lieu, nous avons examiné les propriétés du mélange dissolvant butylamine/cyclohexanone/triton X-100 pour mieux délimiter son domaine d'application à la mesure des activités enzymatiques. Ensuite, nous avons complété l'étude du dosage de la phosphatase alcaline, étant donné son importance dans les laboratoires d'industrie laitière, en mettant au point une épreuve parallèle à la peroxydase. Enfin, nous avons défini des conditions standard de détermination des activités protéolytiques et lactasique naturelles du lait. 


\section{MATERIEL}

Les essais ont été effectués soit sur des échantillons de lait commerciaux pasteurisés soit sur des échantillons de lait cru provenant d'une ferme de la région de Nancy.

Tous les réactifs utilisés sont de la catégorie « pour analyses » ou d'une pureté équivalente. Les réactifs suivants proviennent de Merck, Darmstadt RFA : p-nitrophénylphosphate de sodium (p-NPP), p-phénylène diamine, n-butylamine et o-nitrophényl-galactoside (o-NPG).

Ceux fournis par Prolabo, Paris sont : diéthanolamine (DEA), triéthanolamine (TEA), triton X-100, cyclohexanone, gaïacol, azoture de sodium et diméthylformamide.

Les substrats fournis par Serva, Heidelberg, RFA sont : LAlanine-4-nitroanilide, N-acétyl-L-Alanine-4-nitroanilide, N- $\alpha$ Benzoyl-DLArginine-4-nitroanilide, Glycine-4-nitroanilide, L-Leucine-4-nitroanilide, L-Lysine-4-nitroanilide, N- $\alpha$ Benzoyl-DL-Lysine-4-nitroanilide, L-Phenylalanine-4-nitroanilide, N-glutaryl-L-Phénylalanine-4-nitroanilide, $\quad \mathrm{N}$ acétyl-L-Tyrosine-4-nitroanilide.

Tous les réactifs ont été transférés à l'aide de pipettes réglables à cônes de prélèvement. Les protocoles d'analyse mis au point sont donnés en annexe.

\section{RESULTATS}

\section{Propriétés et domaine d'application du mélange $\mathbf{n}$-butylamine/ cyclohexanone/triton X-100 (B.C.T.)}

Pour la détermination directe par spectrométrie d'absorption moléculaire des activités enzymatiques dans le lait, le mélange dissolvant doit : mesure ;

- présenter une faible extinction à la longueur d'onde de la

- dissoudre les protéines et la matière grasse sans hydrolyser le substrat de l'enzyme ;

- diluer le moins possible l'échantillon.

Linden et Paquet (1981) utilisent le mélange n-butylamine/cyclohexanone/triton X-100. La proportion des constituants est indiquée dans le tableau 1.

Pour le lait, ce mélange dissolvant permet, après un maintien au maximum de 2 min à $37^{\circ} \mathrm{C}$ de rendre l'échantillon transparent sans trop le diluer (dilution au 1/8). Les échantillons de lait qui ont subi des traitements thermiques prononcés peuvent nécessiter un séjour légèrement plus long à $37^{\circ} \mathrm{C}$. 
TABLEAU 1 - TABLE 1

Composition du mélange dissolvant standard

Composition of standard solvent mixtures

\begin{tabular}{l|c|c|c|c}
\hline & \multicolumn{3}{|c|}{ Lait } & \\
\cline { 2 - 3 } & entier & demi-écrémé & écrémé & \\
\cline { 2 - 3 } n-Butylamine & 2 & 2 & 2 & 0,25 \\
Cyclohexanone & 1 & 0,5 & 0 & 1 \\
Triton X-100 & 1 & 1 & 1 & 1 \\
\hline
\end{tabular}

Selon les types de substrats employés, des quantités variables d'acide chlorhydrique concentré doivent être ajoutées au mélange.

Pour la crème, la dissolution est plus délicate à obtenir ; on réussit cependant à " solubiliser » la crème à $45 \%$ de matière grasse en ajoutant séparément les composants du mélange dissolvant et en maintenant le mélange réactionnel à $37^{\circ} \mathrm{C}$ jusqu'à la lecture au spectromètre.

Enfin, on peut noter qu'une diminution de la teneur en matière grasse de l'échantillon permet de réduire la fraction de cyclohexanone dans le mélange.

L'extinction de ce solvant mixte est très faible entre 400 et $800 \mathrm{~nm}$. A $410 \mathrm{~nm}$, ce dernier présente, pour $1 \mathrm{~cm}$ de trajet optique, une extinction égale à 0,06 ; elle devient élevée à $310 \mathrm{~nm}$.

Le $\mathrm{pH}$ apparent du mélange ternaire B.C.T. est égal à 12,2. Il peut être abaissé en ajoutant des quantités croissantes d'acide chlorhydrique. En revanche, le temps nécessaire à une dissolution totale de l'échantillon augmente avec la quantité d'acide ajoutée.

Le mélange lait + B.C.T. doit en outre être stable suffisamment longtemps pour permettre la lecture de grandes séries d'échantillons. A $37^{\circ} \mathrm{C}$, le milieu est stable au moins $30 \mathrm{~min}$. Ensuite, on observe l'apparition d'un léger trouble qui se traduit par une floculation irréversible déjà signalée par Bosset, Blanc et Plattner (1977 a).

Ainsi, l'étude du spectre d'extinction et de la zone de $\mathrm{pH}$ d'utilisation du mélange dissolvant B.C.T. montre que son emploi pour le dosage des activités enzymatiques peut s'appliquer aux substrats susceptibles de libérer un produit de réaction qui absorbe entre 400 à $800 \mathrm{~nm}$ et qui reste suffisamment stable à $\mathrm{pH} 10-11$ pour permettre sa lecture au spectromètre. 


\section{Contrôle de l'activité de la phosphatase alcaline et de la peroxydase du lait après traitement thermique}

\subsection{Phosphatase alcaline (E.C.3.1.3.1)}

En annexe 1, nous proposons un protocole opératoire pour la détermination de l'activité de la phosphatase alcaline dans le lait et quelques produits laitiers (Linden et Paquet, 1981) dans lequel le mélange dissolvant B.C.T. sert également de solution d'arrêt de la réaction enzymatique.

Cette méthode est simple, rapide, fiable et très sensible (tab. 2) puisque le coefficient de variation est voisin de $3 \%$ et qu'une incubation de 20 min permet de détecter un ajout de $0,1 \%$ de lait cru à un lait pasteurisé.

\section{TABLEAU 2 - TABLE 2}

Répétabilité des méthodes proposées pour la détermination de l'activité phosphatasique, protéinasique et $\beta$-D-galactosidasique du lait entier

Repetability of proposed methods of phosphatasic, proteinasic and $\beta$-D-galactosidasic activities determination in whole milk

\begin{tabular}{|c|c|c|c|}
\hline & \multicolumn{3}{|c|}{ Activités } \\
\hline & $\begin{array}{c}\text { Phosphatase } \\
\text { alcaline }\end{array}$ & Protéinases & $\begin{array}{l}\beta \text {-D-galacto- } \\
\text { sidase }\end{array}$ \\
\hline Nombre d'essais (n) & 40 & 32 & 31 \\
\hline Moyenne $(\bar{X})$ & $25,7 \cdot 10^{-2}$ (a) & 79,3 (b) & $2,1 \quad$ (c) \\
\hline Ecart-type (S) & $0,79 \cdot 10^{-2}$ (a) & 3,94 (b) & $0,13(\mathrm{c})$ \\
\hline Coefficient de variation (C.V.) $\%$ & 3,08 & 4,97 & 6,56 \\
\hline \multicolumn{4}{|c|}{$\begin{array}{l}\text { (a) } \mu \mathrm{mol} \cdot \mathrm{ml}^{-1} \text { p.nitrophénol libéré en } 60 \mathrm{~min} \text {. } \\
\text { (b) } \mu \mathrm{mol} \cdot \mathrm{ml}^{-1} \text { 4-nitroanilide libéré en } 24 \mathrm{~h} \text {. } \\
\text { (c) } \mu \mathrm{mol} \cdot \mathrm{ml}^{-1} \mathrm{o} \text {-nitrophénol libéré en } 24 \mathrm{~h} \text {. }\end{array}$} \\
\hline
\end{tabular}

\subsection{Peroxydase (E.C.1.11.1.7)}

Etant donné l'importance du test à la peroxydase dans l'industrie laitière, nous proposons en annexe 2 pour la mise en évidence de l'activité peroxydasique un protocole apparenté au dosage de la phosphatase.

En utilisant un mélange équimoléculaire de gaïacol et de pphénylène-diamine, notre protocole permet de détecter jusqu'à $0,01 \%$ 
de lait cru dans du lait stérilisé (tab. 3). C'est donc un abaissement appréciable des seuils de détection par rapport aux méthodes habituelles.

TABLEAU 3 - TABLE 3

Sensibilité du dosage de la peroxydase dans le lait stérilisé contenant du lait cru

Sensibility of peroxydasic activity determination in sterilized milk containing raw milk

\begin{tabular}{l|c}
\hline & \multicolumn{1}{c}{$470 \mathrm{~nm}$} \\
& Extinction $1 \mathrm{~cm}$ \\
\hline Lait stérilisé & 0,120 \\
Lait stérilisé $+0,5 \%$ de lait cru & 0,246 \\
Lait stérilisé $+0,1 \%$ de lait cru & 0,200 \\
Lait stérilisé $+0,02 \%$ de lait cru & 0,175 \\
Lait stérilisé $+0,01 \%$ de lait cru & 0,166 \\
\hline
\end{tabular}

\section{Détermination de l'activité des protéinases et de la $\beta$-galacto- sidase dans le lait}

\subsection{Activité des protéinases du lait (E.C.3.4.21.X)}

Pour mesurer l'activité protéolytique du lait, plusieurs méthodes ont été employées (Humbert et Alais, 1979; Reimerdes, Petersen et Kielwein, 1979). L'activité protéolytique de type trypsique est mise en évidence par l'emploi de différents substrats de synthèse, appartenant à la série des 4-nitroanilides. Les conditions standard sont définies dans l'annexe 3.

La cinétique, pour tous les substrats de synthèse hydrolysés, est linéaire au moins durant $4 \mathrm{~h}$ et l'activité des échantillons de laits crus prélevés stérilement que nous avons testés dans des conditions aseptiques est suffisamment élevée pour permettre une mesure correcte après un tel laps de temps.

Dans le tableau 2, nous avons déterminé la répétabilité de la méthode avec le substrat L-Alanine-4-nitroanilide ; le coefficient de variation est de $5 \%$.

\subsection{Activité de la $\beta$-D-galactosidase (E.C.3.2.1.23)}

Pour mesurer l'activité lactasique, on utilise l'o-nitrophényl-galactopyranoside (o-NPG). Les conditions opératoires sont consignées dans l'annexe 4. Relevons que nous avons préféré le tampon tris- $\mathrm{HCl} 0,1$ 
mol/1, pH 7 au tampon phosphate avec lequel nous avons observé des difficultés de dissolution du lait par le mélange standard.

L'activité $\beta$-D-galactosidasique naturelle de certains échantillons de lait est très faible. Une période d'incubation pouvant aller jusqu'à $96 \mathrm{~h}$ peut être nécessaire pour permettre une mesure correcte. Comme pour les protocoles précédents, la répétabilité de la méthode est satisfaisante (tab. 2). Avec un échantillon de lait entier à activité faible, nous avons obtenu un coefficient de variation de $6,5 \%$.

\section{DISCUSSION ET CONCLUSION}

La mise au point du solvant mixte n-butylamine/cyclohexanone/ triton X-100 (2:1:1) présente de nombreux avantages, notamment celui de peu diluer l'échantillon (lait, crème, etc.) et celui de dissoudre très rapidement tous les constituants colloïdaux de ce dernier.

La faible extinction du mélange entre 400 et $800 \mathrm{~nm}$, donc pratiquement dans tout le spectre visible offre de larges possibilités de dosages photométriques. Dans nos conditions d'utilisation, le mélange B.C.T. garde son pouvoir dissolvant entre $\mathrm{pH} 12,2$ et 9 .

Les résultats ont montré que ce solvant mixte permet la détermination directe d'activités enzymatiques dans le lait telles celles de la phosphatase alcaline, de la peroxydase, des protéinases et de la $\beta$-D-galactosidase, sans interférer avec la réaction enzymatique proprement dite. Le produit coloré de la réaction est stable pendant un laps de temps suffisant pour permettre une mesure dans de bonnes conditions. Des tests de répétabilité ont montré chaque fois que les protocoles proposés satisfont aux conditions requises pour un laboratoire de contrôle. En outre, pour des épreuves à la phosphatase et à la peroxydase, cette méthode permet un accroissement appréciable de la sensibilité par rapport aux méthodes officielles connues (Linden et Paquet, 1981). De plus, les méthodes proposées sont simples et rapides. Elles ne nécessitent en effet que l'emploi de deux mélanges de réactifs, à savoir la solution substrat-tampon et le mélange dissolvant.

On peut envisager l'utilisation de ces méthodes en analyse semiquantitative sous forme de coffrets équipés d'un comparateur (Linden, 1981). Elles présenteraient ainsi tous les avantages des microtests commercialisés (Monget et Laviolette, 1978) tout en offrant une bien meilleure sensibilité.

\section{Remerciements}

Nous remercions M. le Pr C. Alais pour ses précieux conseils lors de la réalisation de ce travail et la rédaction de ce manuscrit.

Reçu pour publication en février 1982. 


\section{ANNEXES}

\section{DETERMINATION DES ACTIVITES ENZYMATIOUES}

\section{ANNEXE I \\ 1. Principe \\ PHOSPHATASE ALCALINE \\ La phosphatase alcaline (E.C.3.1.3.1.) hydrolyse son substrat, le p-nitro- phénylphosphate disodique. La libération du p-nitrophénol est suivie par spectrométrie après dissolution totale de tous les composants du milieu réactionnel par le mélange dissolvant n-butylamine/cyclo- hexanone/triton X-100.}

\section{Réactifs}

2.1. Solution aqueuse tampon de diéthanolamine/ $\mathrm{HCl} 1 \mathrm{~mol} / \mathrm{l}, \mathrm{pH}$ 10,6.

2.2. Substrat : p-nitrophénylphosphate $5.10^{-3} \mathrm{~mol} / \mathrm{l}$ dans la solution tampon 2.1.

2.3. Mélange dissolvant à $\mathrm{pH}$ apparent de 11,2 :

- 2 vol. n-butylamine.

-1 vol. cyclohexanone.

- 1 vol. triton X-100.

\section{Mode opératoire}

3.1. Introduire dans un tube à essai :

- $0,5 \mathrm{ml}$ de lait (ou $0,5 \mathrm{~g}$ de crème).

$-1,5 \mathrm{ml}$ de solution substrat (2.2).

3.2. Agiter et laisser incuber à $37^{\circ} \mathrm{C}$ pendant 15 à $60 \mathrm{~min}$.

3.3. Ajouter $2 \mathrm{ml}$ de mélange dissolvant 2.3.

3.4. Agiter et laisser réagir $2 \mathrm{~min}$ à $37^{\circ} \mathrm{C}$.

3.5. Lire l'extinction de la solution à $410 \mathrm{~nm}$, à $37^{\circ} \mathrm{C}$ avant $20 \mathrm{~min}$.

\section{Principe}

\section{ANNEXE II}

\section{PEROXYDASE}

Cette méthode repose sur la décomposition de l'eau oxygénée par la peroxydase (E.C.1.11.1.7) et fixation de l'oxygène atomique sur deux accepteurs. La mesure du colorant ainsi produit se fait par spectrométrie après dissolution totale des divers composants du milieu réactionnel par le mélange dissolvant n-butylamine/cyclohexanone/ triton X-100.

\section{Réactifs}

2.1. Solution aqueuse saturée de gaïacol (environ $21 \%$ ).

2.2. Solution aqueuse de p-phénylènediamine (PDA) à $2 \%$.

2.3. Eau oxygénée à $10 \% \mathrm{v} / \mathrm{v}$. 
2.4. Mélange dissolvant à $\mathrm{pH}$ apparent de 11,2 :

- 2 vol. n-butylamine.

-1 vol. cyclohexanone.

-1 vol. triton $\mathrm{X}-100$.

\section{Mode opératoire}

3.1. Introduire dans un tube à essai :

$-1 \mathrm{ml}$ de lait.

$-1 \mathrm{ml}$ de solution de gaïacol (2.1.).

- $1 \mathrm{ml}$ de solution PDA (2.2.).

- 0,5 $\mathrm{ml}$ d'eau oxygénée (2.3.).

3.2. Ne pas agiter. Observer qualitativement l'apparition éventuelle d'une teinte violette mettant en évidence la présence de peroxydase.

3.3. Ajouter $2 \mathrm{ml}$ de mélange dissolvant (2.4.).

3.4. Agiter et laisser réagir $2 \mathrm{~min}$ à $37^{\circ} \mathrm{C}$.

3.5. Lire l'extinction de la solution à $470 \mathrm{~nm}$, à $37^{\circ} \mathrm{C}$ avant $20 \mathrm{~min}$.

\section{Principe}

\section{ANNEXE III}

\section{PROTEINASES}

L'activité protéolytique (E.C.3.4.21.X) est mise en évidence par l'emploi de différents substrats de synthèse, appartenant à la série des 4-nitroanilides, qui sont hydrolysés au cours de l'incubation. La mesure se fait par spectrométrie après dissolution totale du milieu réactionnel par le mélange dissolvant n-butylamine/cyclohexanone/ triton X-100.

\section{Réactifs}

2.1. Solution tampon $\mathrm{pH} 8$ :

- triéthanolamine $/ \mathrm{HCl} \quad 0,3 \mathrm{~mol} / \mathrm{l}$.

- azide de sodium $0,02 \%$.

2.2. Diméthylformamide.

2.3. Substrats : dérivés 4-nitroanilide d'acides aminés ou de peptides (voir le chapitre Matériel).

Le substrat est préalablement dissous dans la solution (2.2.) par un léger chauffage, puis dilué dans la solution tampon (2.1.) de manière à obtenir une concentration finale de $5.10^{-3} \mathrm{~mol} / 1$ en substrat et de $10 \%$ en diméthylformamide.

2.4. Mélange dissolvant à $\mathrm{pH}$ apparent de 11,2 :

-2 vol. n-butylamine.

-1 vol. cyclohexanone.

-1 vol. triton $\mathrm{X}-100$. 
3. Mode opératoire

3.1. Introduire dans un tube à essai :

$-0,5 \mathrm{ml}$ de lait.

$-1,5 \mathrm{ml}$ de solution substrat (2.3.).

3.2. Agiter et laisser incuber à $37^{\circ} \mathrm{C}$ pendant 8 à $24 \mathrm{~h}$.

3.3. Ajouter $2 \mathrm{ml}$ de mélange dissolvant (2.4.).

3.4. Agiter et laisser 2 min à $37^{\circ} \mathrm{C}$.

3.5. Lire l'extinction de la solution à $410 \mathrm{~nm}$, à $37^{\circ} \mathrm{C}$ avant $20 \mathrm{~min}$.

\section{Principe}

\section{ANNEXE IV}

La $\beta$-D-galactosidase (E.C.3.2.1.23) scinde le lactose en glucose et galactose. Cette activité est mise en évidence par l'emploi comme substrat de l'o-nitrophényl-galactopyranoside qui est hydrolysé en o-nitrophénol et galactose. La mesure se fait par spectrométrie après dissolution totale du milieu réactionnel par le mélange dissolvant n-butylamine/cyclohexanone/triton X-100.

\section{Réactifs}

2.1. Solution tampon à $\mathrm{pH} 7$;

- tris- $\mathrm{HCl} 0,1 \mathrm{~mol} / \mathrm{l}$.

- azide de sodium $0,02 \%$.

2.2. Substrat : o-nitrophénol- $\beta$-galactopyranoside $5.10^{-3} \mathrm{~mol} / \mathrm{l}$ dans la solution tampon (2.1).

2.3. Mélange dissolvant à $\mathrm{pH}$ apparent de 11,2 :

- 2 vol. n-butylamine.

-1 vol. cyclohexanone.

-1 vol. triton $\mathrm{X}-100$.

\section{Mode opératoire}

3.1. Introduire dans un tube à essai :

$-0,5 \mathrm{ml}$ de lait.

$-1,5 \mathrm{ml}$ de solution substrat (2.2.).

3.2. Agiter et laisser incuber à $37^{\circ} \mathrm{C}$ pendant 24 à $96 \mathrm{~h}$.

3.3. Ajouter $2 \mathrm{ml}$ de mélange dissolvant (2.3.).

3.4. Agiter et laisser $2 \mathrm{~min}$ à $37^{\circ} \mathrm{C}$.

3.5. Lire l'extinction du mélange réactionnel à $410 \mathrm{~nm}$, à $37^{\circ} \mathrm{C}$, avant $20 \mathrm{~min}$. 


\section{Bibliographie}

Bosset (J. O.) et Blanc (B. H.) (1977). - Nouvelle méthode simple et économique destinée au dosage de routine des protéines du lait et de quelques produits laitiers. Lait. Rom., 53, 697-699.

Bosset (J. O.), Blanc (B. H.) et Plattner (E.) (1977 a). - La dissolution intégrale du lait entier au moyen de solvants mixtes. I. Etude du mélange dissolvant : eau-hydroxyde de sodium-n-butylamine. Trav. Chim. Aliment. Hyg., 68, 225-239.

Bosset (J.O.), Blanc (B. H.) et Plattner (E.) (1977 b). - La dissolution intégrale du lait entier au moyen de solvants mixtes. II. Etude du mélange dissolvant: eau-hydroxyde de sodium-tétrahydrofurane. Trav. Chim. Aliment. Hyg., 68, 504-512.

Humbert (G.) and Alais (C.) (1979). - Review of the progress of Dairy Science: the milk proteinase system. J. Dairy Res., 46, 559-571.

Linden (G.) (1981). - Méthodes rapides d'évaluation des activités enzymatiques in "Méthodes rapides de contrôles de fabrication dans les industries agricoles et alimentaires. Leur développement et leurs performances ». C.R. Colloque APRIA, Paris, p. 155-162.

Linden (G.) and Paquet (D.) (1981). - Alkaline phosphatase activity in transparent milk and cream. J. Dairy Sci., 64, 568-571.

Monget (D.) et Laviolette (P.) (1978). - Mise au point de microtests " phosphatase alcaline " et " peroxydase " pour le contrôle de la pasteurisation du lait de vache. Lait, 58, 595-605.

Reimerdes (E. H.), Petersen (F.) und Kielwein (G.) (1979). - Milchproteinase-9Proteinasepektren von Caseinmicellen, Milchserum, Rinderblutserum und Pseudomonas fluorescens. Milchwissenschaft, 34, 548-551. 